\title{
CRT-BASED THREE-PRIME RSA WITH IMMUNITY AGAINST HARDWARE FAULT ATTACK
}

\author{
Yonghong Yang, Z. Abid and Wei Wang \\ Dept. of Electrical \& Computer Engineering, the University of Western Ontario \\ 1151 Richmond St, London, Ontario, Canada, N6A $5 B 9$ \\ Email:yyang62@uwo.ca,zeabid@eng.uwo.ca,wwang@eng.uwo.ca
}

\begin{abstract}
In this paper, we carry out the study of the Chinese Remainder Theorem based three-prime RSA cryptosystem. The hardware fault attack on three-prime RSA cryptosystem is analyzed and it is proven that the three-prime RSA is more difficult to be broken than two-prime RSA by the hardware fault attack. Then, Shamir's checking procedure is extended from two-prime to three-prime RSA to increase its immunity against such attack. Finally an immune method for three-prime RSA without checking procedure is proposed in this paper, which is more efficient than the previous methods. It is expected that this proposed system will play an important role in the future cryptography applications.
\end{abstract}

\section{Introduction}

Since the RSA cryptography was proposed in 1978 [1], it has been widely used in security communications. The calculations in RSA involve large time complexity and many implementation resources. Chinese Remainder Theorem (CRT) is often used in multi-prime cryptosystem [2] to speed up the calculations in RSA [3].

After the analysis of the hardware fault attack on the conventional three-prime CRT-based RSA, we propose an immune three-prime RSA system against such attack with more efficient calculations in this paper. Compared to the conventional CRT-based RSA, the proposed three-prime RSA utilizes an additional summing term on the basis of the conventional CRT-based RSA other than a product term, which is used in the two methods in [4]. It also uses three smaller modular exponentiation computations instead of the large one in [4]. The new system ulteriorly simplifies the calculations by avoiding the division calculation needed in the second protocol in [4]. Thus, the new method provides higher operation speed than the two methods in [4].

The rest of the paper is organized as follows. The architecture of three-prime CRT-based RSA is described in section 2. Section 3 is dedicated to the attack analysis of the system. In section 4, Shamir's checking procedure is extended to three-prime RSA to increase its immunity. An immune three-prime RSA system without checking procedure, which has more efficient calculations than existing methods [4], is proposed in section 5. Then, both the conventional and the proposed three-prime RSA are implemented on FPGA chip in section 6 for comparison purpose. Finally, the paper is concluded in section 7.

\section{Basic RSA cryptosystems}

\subsection{General RSA cryptosystem}

One process in RSA system is encryption/decryption and the other is signing/signature verification. The encryption/decryption process is as follows. By using the public key $e$, a message $m$, which is smaller than the public modulus $n$, can be encrypted as $c=m^{e} \bmod n$. The message $m$ can be recovered from $c$ by using the secret key $d$ as $m=c^{d} \bmod n$. In the signing/signature verification process, the secret key $d$ is used to obtain the signature of the message $m$ as $s=m^{d}(\bmod n) . m$ can be recovered from $s$ by the public key $e$ as $m=s^{e} \bmod n$. In this paper, our study concentrates on the signing process of the three-prime RSA system.

\subsection{Structure of CRT-based three-prime RSA}

In three-prime cryptosystem, $p, q$ and $r$ are three large prime numbers such that $n=p \cdot q \cdot r$. The signing process can be computed more efficiently by applying CRT as follows:

1) $m_{p}=m \bmod p, m_{q}=m \bmod q$ and $m_{r}=m \bmod r$ are computed first. 
2). $s_{p}=m_{p}{ }^{d p} \bmod p \quad, \quad s_{q}=m_{q}{ }^{d_{q}} \bmod q \quad$ and $s_{r}=m_{r}{ }^{d_{r}} \bmod r$ are computed next, where $d_{p}=d \bmod (p-1) \quad, \quad d_{q}=d \bmod (q-1) \quad$ and $d_{r}=d \bmod (r-1)$ respectively.

3) Finally, $s$ can be obtained using CRT as:

$$
s=C R T\left(s_{p}, s_{q}, s_{r}\right) .
$$

The functional diagram for the signing process of three-prime CRT-based RSA, as described above, is shown in Fig.1.



Fig. 1. Functional diagram of the signing process

The three-prime RSA uses three channels with numbers of smaller wordlengths, while two -prime RSA uses two channels with numbers of larger wordlengths. Thus, the three-prime RSA provides higher operation speed than the two-prime case.

\section{Attack on three-prime RSA}

There is no previous work regarding the analysis of the hardware fault attack on three-prime CRT -based RSA. This work is carried out in this paper. The hardware fault attack on two-prime RSA is described here first.

For the CRT-based RSA cryptosystem, the attacker is able to purposely induce some types of hardware fault into the system. Then the affected computation values may be used to factor the public modulus of the system [5]. This is called hardware fault attack.

A faulty signature $s^{\prime}$ will be computed based on $s_{p}^{\prime}$ (the faulty value of $s_{p}$ ), which is induced by some error due to the interference of the attacker, and the fault-free $s_{q}$. After intercepting the faulty signature $s^{\prime}$, the attacker will be able to factor the modulus of the two-prime RSA system by computing $q=\operatorname{gcd}\left(\left(s^{\prime e}-m\right) \bmod n, n\right)$ and $p=n / q$ [4].

For three-prime RSA cryptosystem with $n=p \cdot q \cdot r$, the attack involves two faulty signatures $s_{1}^{\prime}$ and $s_{2}^{\prime}$, which are computed based on different faulty results of $s_{i}$ ( $i$ represents $p, q$ or $r$ ) respectively.

Proposition 1: The attacker can factor modulus $n$, only after intercepting two faulty signatures $s_{1}^{\prime}$ and $s_{2}^{\prime}$, by using the hardware fault attack as follows:

$$
\begin{aligned}
& q r=\operatorname{gcd}\left(\left(s_{1}^{\prime e}-m\right) \bmod n, n\right), \\
& p r=\operatorname{gcd}\left(\left(s_{2}^{\prime e}-m\right) \bmod n, n\right), \\
& p=n / q r=n / \operatorname{gcd}\left(\left(s_{1}^{\prime e}-m\right) \bmod n, n\right), \\
& q=n / p r=n / \operatorname{gcd}\left(\left(s_{2}^{\prime e}-m\right) \bmod n, n\right), \\
& r=n / p q .
\end{aligned}
$$

Proof is omitted here due to space limit. In order to factor the three-prime CRT-based RSA cryptosystem using hardware fault attack, two faulty signatures and the corresponding factorization calculations are needed. Thus, three-prime CRT-based RSA cryptosystem brings more difficulty for the attacker compared to two-prime RSA, which requires only one faulty signature and the corresponding calculations to be factored.

\section{Proposed extension of Shamir's countermeasure}

In order to counteract the above hardware fault attack, Shamir presented a simple countermeasure for two-prime RSA using the checking procedure [5]. In the original checking method, the following definitions are used.

$$
\begin{aligned}
& s_{p t}=m^{d \bmod \phi(p t)} \bmod (p t) \text { and } \\
& s_{q t}=m^{d \bmod \phi(q t)} \bmod (q t),
\end{aligned}
$$

where $\phi$ is Euler's totient function and $t$ is a random integer.

Here we extend the countermeasure to three-prime RSA. For three-prime RSA cryptosystem, $s_{p t}, s_{q t}$ are calculated as explained above and an additional $s_{r t}$ is computed as $s_{r t}=m^{d \bmod \phi(r t)} \bmod (r t)$ first. If $s_{p t} \bmod t=s_{q t} \bmod t=s_{r t} \bmod t$, then the signature $s$ calculated by $C R T\left(s_{p t} \bmod p, s_{q t} \bmod q, s_{r t} \bmod r\right)$ is 
regarded as error-free. Otherwise, the signature should be recalculated.

Proposition 2: For three-prime RSA cryptosys tem, the probability of the checking procedure to produce an undetectable error is $p_{e} /(t-1)^{2}$, where $p_{e}$ is the probability for the system to get wrong computation results of $s_{p t}, s_{q t}$ and $s_{r t}$.

Proof: When no error occurs in the computations, $s_{p t} \bmod t=s_{q t} \bmod t=s_{r t} \bmod t=m^{d} \bmod t$. And the probability for $s_{p t} \bmod t=s_{q t} \bmod t=s_{r t} \bmod t$ when error occurs in the computation of $s_{p t}, s_{q t}$ and $s_{r t}$ is $1 /(t-1)^{2}$. Taking $p_{e}$ into consideration, we get the probability for the system to take unequivalent $s_{p t} \bmod t \quad, \quad s_{q t} \bmod t \quad$ and $s_{r t} \bmod t$ for $s_{p t} \bmod t=s_{q t} \bmod t=s_{r t} \bmod t$ is $p_{e} /(t-1)^{2}$. Q.E.D

The use of the checking procedure before the signatures are sent out of the system is effective to counteract hardware fault attack to some extent. However, the checking procedure itself depends on the reliability of a decision procedure whose result may be easily modified [4]. Thus, countermeasures without checking procedure are desirable for the cryptosystem.

\section{Proposed immune method}

Two methods without checking procedure were proposed in [4] to improve the immunity against hardware fault attack for two-prime cryptosystem. These two methods achieve the desired performance by influencing the computation of $s_{q}$ or the overall computation of $s$ when an error occurs in the computation of $s_{p}$. This property also applies when a faulty $s_{q}$ is produced.

In these two methods, the signature is calculated using $s=\left(C R T\left(s_{p}, s_{q}\right) \times\left(\tilde{m}^{t}\right)\right)(\bmod n) \quad$ instead of $\operatorname{CRT}\left(s_{p}, s_{q}\right)$, where $\tilde{m}$ is calculated based on $s_{p}$ and $s_{q}$. The additional product term $\tilde{m}$ ensures that the public modulus $n$ will not be factored even the faulty signature is intercepted by the attacker.

A new immune three-prime RSA without checking procedure is proposed as follows.

Step 1: $\left(e_{t}, d_{t}\right)$ is chosen where $d_{t}=d-t . t$ and $e_{t} \equiv d_{t}^{-1}(\bmod \phi(n))$ both are small integers and $e_{t}$ is larger than $t$.

Step 2:

$$
\begin{aligned}
& k_{p}=\lfloor m / p\rfloor, k_{q}=\lfloor m / q\rfloor \text { and } k_{r}=\lfloor m / r\rfloor, \\
& s_{i t}=(m \bmod i)^{d_{t} \bmod (i-1)} \bmod i, \\
& s_{i}=s_{i t}^{t} \bmod i,
\end{aligned}
$$

where $i$ represents $p, q$ and $r$, are calculated.

Step 3: The signature $s$ can be obtained by adding a summing term to the conventional CRT operation as:

$\left.s=\left(C R T\left(s_{p}, s_{q}, s_{r}\right)+\tilde{m}\right)\right) \bmod n$,

where

$$
\begin{aligned}
\tilde{m}= & \left(\left(s_{p} \cdot s_{p t}^{e_{t}-t} \bmod p+k_{p} \cdot p\right)+\left(s_{q} \cdot s_{q t}^{e_{t}-t} \bmod q+k_{q} \cdot q\right)\right. \\
& \left.-2 \times\left(s_{r} \cdot s_{r t}^{e_{t}-t} \bmod r+k_{r} \cdot r\right)\right) \times n .
\end{aligned}
$$

Now we prove that the value of the signature calculated as above equals to that calculated by conventional CRT.

Theorem 1: $\left.\left(\operatorname{CRT}\left(s_{p}, s_{q}, s_{r}\right)+\tilde{m}\right)\right)\left.\right|_{n}=\operatorname{CRT}\left(s_{p}, s_{q}, s_{r}\right)$

if no error occurs in the calculation of equation (1).

Proof:

When no error occurs in the computation of $s_{p t}, s_{q t}$ and $s_{r t}$, then

$$
s_{p} \cdot s_{p t}^{e_{t}-t} \bmod p+k_{p} \cdot p=m .
$$

Similarly,

$$
\begin{aligned}
& s_{q} \cdot s_{q t}^{e_{t}-t} \bmod q+k_{q} \cdot q=m, \\
& s_{r} \cdot s_{r t}^{e_{t}-t} \bmod r+k_{r} \cdot r=m
\end{aligned}
$$

Thus,

$$
\begin{aligned}
\tilde{m} & =(m+m-2 \times m) \times n=0, \text { which results in } \\
s & =\left(C R T\left(s_{p}, s_{q}, s_{r}\right)+\tilde{m}\right)(\bmod n) \\
& =C R T\left(s_{p}, s_{q}, s_{r}\right) .
\end{aligned}
$$

Theorem 2: If two faulty signatures $s_{1}^{\prime}=\left(C R T\left(s_{p}^{\prime}, s_{q}, s_{r}\right)+\tilde{m}\right)(\bmod n) \quad$ and $s_{2}^{\prime}=\left(C R T\left(s_{p}, s_{q}^{\prime}, s_{r}\right)+\tilde{m}\right)(\bmod n) \quad$ are intercepted from the three-prime RSA cryptosystem, then the complexity of factoring $n$ by computing

$$
\begin{gathered}
q r=\operatorname{gcd}\left(\left(C R T\left(s_{p}^{\prime}, s_{q}, s_{r}\right)^{e}-m\right) \bmod n, n\right) \\
p r=\operatorname{gcd}\left(\left(C R T\left(s_{p}, s_{q}^{\prime}, s_{r}\right)^{e}-m\right) \bmod n, n\right)
\end{gathered}
$$

is $O(n)$.

Proof:

The result can be obtained similarly as Theorem 3 in [4]. The detailed proof is omitted here due to space limit.

Q.E.D

If CRT-1 and CRT-2 protocols are extended to three-prime RSA, the complexity to factor such systems when two faulty signatures are intercepted is also $O(n)$. Thus, the proposed RSA system bears 
similar immunity against attack as these two protocols. We can see that the new method provides higher operation speed than these two methods in the following.

When CRT-1 protocol is extended to three-prime RSA cryptosystem, the values of $s_{p}, s_{q}$ and $s_{r}$ can only be calculated in serial because the computation of $s_{q}$ depends on the value of $s_{p}$, while the computation of $s_{r}$ depends on the value of $s_{q}$. Thus, CRT-1 protocol has much slower speed than CRT-2 protocol and our proposed method where these three calculations are executed in parallel.

In spite of the similar calculations in the CRT-2 protocol, if extended to three-prime case, and our proposed method. The main difference in the calculations of these two methods are listed below.

First, the calculation of $\tilde{m}^{t} \bmod n$ is needed in CRT -2 protocol, where $\tilde{m}$ is a number smaller than $n$. While we calculate $s_{p t}^{t} \bmod p, s_{q t}^{t} \bmod q$, and $s_{r t}^{t} \bmod r$ in parallel instead, where $s_{p t}, s_{q t}$ and $s_{r t}$ are smaller than $p, q$ and $r$ respectively. Moreover, a division by 3 computation is needed in CRT-2 protocol, whereas it is avoided in our proposed method. Thus, the proposed method provides higher speed than CRT -2 protocol [4]. The simple architecture for the proposed method is shown as Fig. 2.

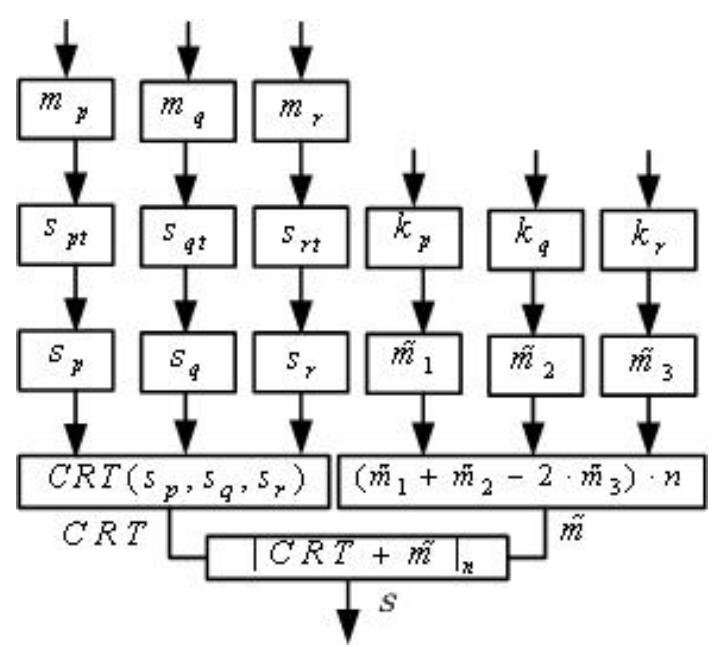

Fig. 2. Three-prime cryptosystem with immunity against hardware fault attack

\section{Implementation}

Both the conventional and the proposed CRT-based three-prime RSA cryptosystems are implemented on the FPGA chip, while the xcv1000-bg560 device is chosen for the implementation. The results of the implemented RSA cryptosystems with 32-bit modulus are shown in Tab 1. $d_{t}$ is assumed to be a 3-bit digit in the implementation.

TABLE 1:

Implementation results for three-prime RSA

\begin{tabular}{|l|c|c|}
\hline & Gates & Clock cycles \\
\hline $\begin{array}{l}\text { conventional } \\
\text { CRT-based RSA }\end{array}$ & $76 \mathrm{k}$ & 411 \\
\hline Proposed RSA & $82 \mathrm{k}$ & 457 \\
\hline
\end{tabular}

The above table shows that the proposed RSA system uses only a few more resources than the conventional CRT-based three-prime RSA. It also only needs a few more clock cycles to finish an encryption/signature operation with much better immunity against the hardware fault attack.

\section{Conclusion}

In this paper, a non-checking immunity method has been proposed for three-prime RSA cryptosystem against hardware fault attack. The proposed method revises the calculation expression of the signature and replaces the larger modular exponentiation operation required by the previous methods [4] with smaller ones. The proposed three-prime RSA provides higher operation speed than the two methods in [4]. The proposed system has been implemented using FPGA technology. Compared to the conventional CRT-based RSA, the new system offers much better immunity while requiring similar hardware resources and providing similar speed.

\section{References}

[1] R. Rivest, A. Shamir, and L. Adleman, “ A Method for Obtaining Digital Signatures and Public-Key Cryptosystems," Communications of the ACM, vol. 21, pp. 120-126, Feb. 1978.

[2] RSA Laboratories, "PKCS \#1 v2.0 Amendment 1: Multi-Prime RSA," July 2000, online: ftp://ftp.rsasecurity.com/pub/pkcs/pkcs-1/pkcs-1v2-0a1. pdf.

[3] C. Wu, J. Hong and C. Wu " RSA Cryptosystem Design Based on the Chinese Remainder Theorem," Proceedings of the ASP-DAC 2001, Las Vegas, USA, Jan-Feb. 2001, pp:391 - 395.

[4] S. Yen, S. Kim, S. Lim and S. Moon, " RSA Speedup with Chinese Remainder Theorem Immune against Hardware Fault Attack," IEEE Transactions on computers, vol. 52, pp 461-472, April 2003.

[5] A. Shamir, "How to Check Modular Exponentiation," EUROCRYPT '97, Konstanz, German, May 1997. 